\title{
The Molecular Mechanism of Rhein in Diabetic Nephropathy
}

\author{
Cong-Cong Zeng, ${ }^{1}$ Xi Liu, ${ }^{1}$ Guo-Rong Chen, ${ }^{2}$ Qian-Jia Wu, ${ }^{1}$ Wang-Wang Liu, ${ }^{2}$ \\ Hai-Ying Luo, ${ }^{1}$ and Jin-Guo Cheng ${ }^{1}$ \\ ${ }^{1}$ Department of Nephrology, The Wenzhou Traditional Chinese Medicine Affiliated Hospital of Zhejiang Chinese Medical University, \\ Wenzhou, Zhejiang 325000, China \\ ${ }^{2}$ Department of Pathology, The First Affiliated Hospital of Wenzhou Medical University, Wenzhou, Zhejiang 325015, China
}

Correspondence should be addressed to Jin-Guo Cheng; chengjinguo1963@yahoo.com

Received 15 July 2014; Revised 2 September 2014; Accepted 3 September 2014; Published 11 November 2014

Academic Editor: Mohamed Eddouks

Copyright (c) 2014 Cong-Cong Zeng et al. This is an open access article distributed under the Creative Commons Attribution License, which permits unrestricted use, distribution, and reproduction in any medium, provided the original work is properly cited.

Diabetic nephropathy $(\mathrm{DN})$ is characterized by unclear pathogenesis. Recent medical data shows that the incidence of DN rises year by year. Rhein is the main compositions of rhubarb, a traditional Chinese medicinal plant, which plays an active role in kidney protection. The prophylaxis and phytotherapeutic effects of rhein are due to its anti-inflammatory and antifibrosis properties. Here, we shed light on the renal protective role of rhein in diabetes mellitus (DM) with a particular focus on the molecular basis of this effect.

\section{Introduction}

Diabetes mellitus (DM) shares a significant medical burden all over the world with its high incidence and numerous complications [1]. Poorly controlled blood glucose leads to the occurrence and development of complications in patients with DM [2]. As one of the most serious diabetic microvascular diseases, diabetic nephropathy (DN) is the leading cause of end-stage renal failure $[3,4]$. However, we have not found the exact pathogenesis of diabetic nephropathy, bringing certain difficulty to cure. Therefore, to explore the exact and feasible drug is current research hotspot and trouble in medicine.

In 1980s, rhubarb, a traditional Chinese medicinal herb, was first used to treat the patients who acquired $\mathrm{DN}$ by a Japanese scholar [5]. Hence, ample studies find the major renoprotective components of rhubarb are rhein, emodin, and chrysophanol, especially rhein [6, 7]. Rhein (4,5-dihydroxyl2-carboxylic-9,10-dihydrodiketoanthracene. Figure 1(a)) a free anthraquinone compound isolated from rhubarb, has extensive pharmacological actions, including the antibacterial, antiviral, anti-inflammatory, antiproliferative, and antifibrosis properties [8]. Although rhein has been widely used to treat numerous diseases in model animals [9-12] during recent decades and many studies have also revealed its pharmacological functions and mechanisms, no comprehensive article has yet been showed on DN. In this review, we summarize in vivo and in vitro experiments that suggest that rhein mediates multiple molecular targets implicated in DN.

\section{The Pathogenesis of Diabetic Nephropathy}

The pathophysiological performances of DN include renal hypertrophy, glomerular and tubular basement membranes thickening, mesangial matrix expansion, ultimately renal glomerular fibrosis, and sclerosis [13]. There are a lot of pathogenic mechanisms that (Table 1) can contribute to the development of this disease, such as glucose and lipid metabolism disorders, hemodynamic dysfunctions, abnormally expressed cytokines, oxidatives stress mechanisms, and genetic susceptibility [14].

\section{Hypoglycemic and Hypolipidemic Benefits of Rhein}

Rhein turned out to attenuate the glucose and lipid metabolism disorders. For example, in diet-induced obese mice, 
<smiles>O=C(O)c1cc(O)c2c(c1)C(=O)c1c(O)cccc1C2=O</smiles>

(a)<smiles>NC(=NNC[C@H](N)C(=O)O)NOc1cc(O)c2c(c1)C(=O)c1cccc(O)c1C2=O</smiles>

(b)

FIgURE 1: The chemical structure of rhein (a) and argirein (b).

rhein shows good antihyperglycemic effect and lipidlowering activity [15]. Besides, Zheng et al. use MCGT1 cells, a glucose transporter 1 (GLUT1) transgenic rat mesangial cell line, as a model to mimic mesangial cells in diabetic conditions, to elucidate that rhein can bring down the glucose levels in MCGT1 cells and the expression of GLUT1 by inhibiting the increased activity of the hexosamine pathway [16]. The increased activity of the hexosamine pathway has been considered as a key element involved in the metabolic disturbances of diabetes [17]. The expression of GLUT1 is obviously increased in patients with DN, and its expression intensity correlates with kidney disease severity. Not only so, the excessive expression of GLUT1 may activate hexoseamine pathway, followed by the production of TGF- $\beta 1$. In accordance with the above theory, another study found that rhein markedly downregulates TGF- $\beta 1$, thereby reducing GLUT1 mRNA expression in mesangial cells [18]. Rhein was also found to inhibit peroxisome proliferator-activated receptor gamma (PPAR $\gamma)$ signaling [19] and suppress the expression of sterol regulatory element-binding protein-1c (SREBP-1c) [20], leading to blocking high-fat diet-induced obesity and decreasing fat mass and lower serum cholesterol and LDL cholesterol in the mice, and ameliorating lipid metabolism. In addition, a near research revealed that the mitochondria fission/fusion modulator-dynamin-related protein 1 (Drp1) plays an important role in promoting hyperglycemia-induced apoptosis of $\beta$-cells, while rhein reversed the expression of Drpl and then largely localized at mitochondria in the $\beta$-cells and strongly protected pancreatic $\beta$-cells from hyperglycemia-induced apoptosis [21]. Moreover, rhein has been proved to improve insulin secretory function of pancreatic $\beta$-cells by preservation of $\beta$-cell mass and inhibition of $\beta$-cell apoptosis and enhance the ability of glucose tolerance [22].

\section{Anti-Inflammatory Benefits of Rhein}

Recently, a large body of studies elucidate that inflammatory responses are vital to the pathogenesis of diabetic nephropathy. Rhein, a natural medicine extracted from rhubarb, is proved to have an anti-inflammatory effect in animals and the clinic $[23,24]$. Rhein is characterized by down- regulating proinflammatory cytokines and signal transducers, such as VCAM-1, activator protein (AP-1), NF- $\kappa$ B, MMPs, and MEK/ERK dependent pathways. Linlin Peng's results indicate that rhein could effectively suppress integrinlinked kinase (ILK) expression and regulate abnormal matrix metalloproteinase-9/tissue inhibitor of metalloproteinase1 ratio (MMP-9/TIMP-1) in HK-2 cells and inhibit the progress of epithelial-mesenchymal transition (EMT). These inhibitory effects of rhein are due to the ILK suppression [25].

Also, it is demonstrated that rhein effectively suppresses the overexpression of fibronectin and TGF- $\beta$, thus reducing accumulation of extracellular matrix, protecting renal function, and ameliorating renal histological changes [26, 27]. Furthermore, argirein (Figure 1(b)), a supermolecule derived from chemical modification of rhein by combining with Larginine through hydrogen bond (Figure 1(b)), is effective in suppressing the proinflammatory cytokines contributing to the pathogenesis of DN dependent on both antiinflammatory activity of rhein and the NO offering activity of L-arginine. Argirein attenuates diabetic nephropathy in streptozotocin-injected rats through suppressing upregulated communication molecule Cx43 (connexin 43) but improving the depressed expression of PPAR $\alpha$ remarkably in renal tissue [28].

\section{Antioxidant Benefits of Rhein}

Although the precise mechanism of DN is still ambiguous, oxidative stress has been deemed as a central mediator in promoting the progression of nephropathy in patients who have diabetes. Rhein's unique structure that contains some powerful polar groups, including one carboxyl and two hydroxyls, contributes to its benefits of antioxidant [29]. Excessive production and generation of reactive oxygen species (ROS) induced by sustained hyperglycemia are a crucial contributor underlying the pathogenesis of diabetes associated with macrovascular and microvascular complications including diabetic nephropathy. While Heo et al.s finding [30] that rhein could decrease ROS production and NADPH oxidase p47(phox) activation proves the antioxidant properties of rhein, at the same time, this discovery provides the basis for rhein becoming an antioxidant possessing promising therapeutic potential in disrupting the development of DN. 
TABLE 1: The pathogenesis of DN.

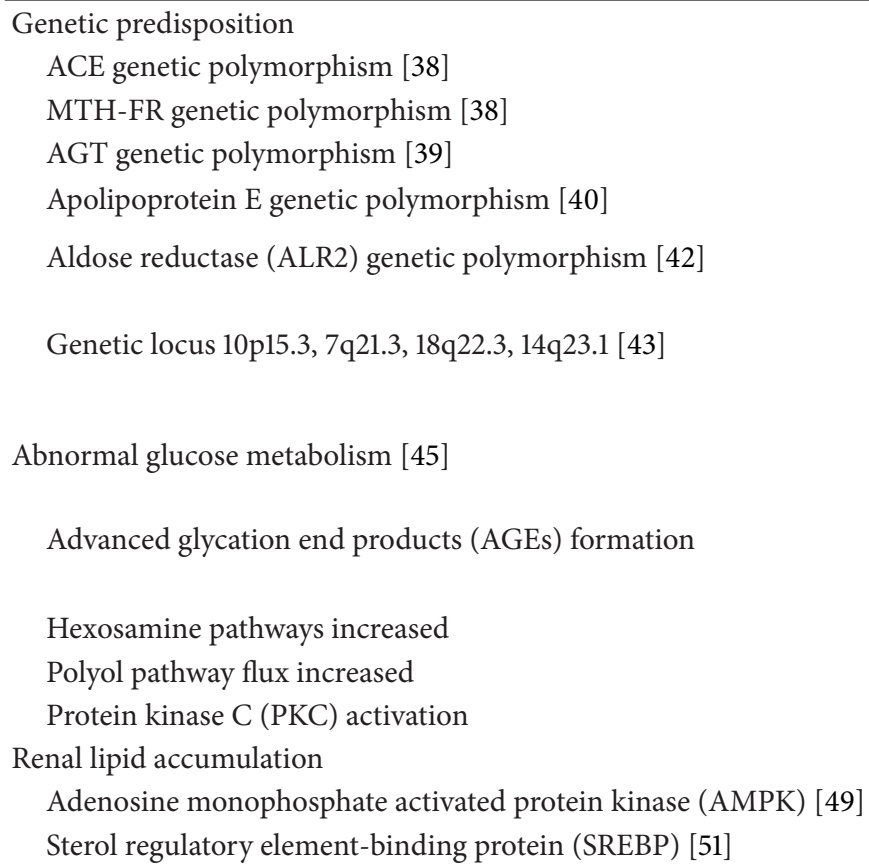

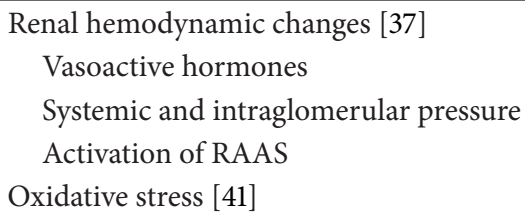

Also, in an experiment which uses hydrogen peroxide $\left(\mathrm{H}_{2} \mathrm{O}_{2}\right)$ to induce injury in human umbilical vein endothelial cells (HUVECs), rhein significantly increased the viability of $\mathrm{H}_{2} \mathrm{O}_{2}$-injured HUVECs by decreasing the malondialdehyde (MDA) and lactate dehydrogenase (LDH) content, increasing the nitric oxide (NO) content and nitrogen oxide synthase (NOS), superoxide dismutase (SOD), and glutathione peroxidase (GSH-PX) activity [31]. In the according article occurring before [28], it simultaneously illuminated that the mRNA and protein distribution of NADPH oxidase (subunits of p22phox, p47phox, and p67phox) in renal tissue may be mitigated by antioxidant activity of argirein.

To sum up, rhein can suppress hyperglycemia, hyperlipidemia, inflammation, and oxidative stress, interacting with multiple molecular targets, therefore improving the pathological performance happening in the progress of diabetic nephropathy (Figure 2).

\section{Pharmacokinetic Studies and Clinical Trials of Rhein}

Because of its beneficial influence on diabetic nephropathy, rhein may be an efficacious antidiabetic nephropathic drug. In order to have more detailed understanding of its safety and bioavailability, many pharmacokinetic studies and clinical trials of rhein have been done. Heo et al. used a physiologically based pharmacokinetic (PBPK) model of rhein to predict human pharmacokinetics. They observed that when it was taken orally by rats, rhein was rapidly absorbed and then was mainly subjected to conjugations such as glucuronidation and sulfation, indicating that phase II \{UDP-glucuronosyltransferase (UGT) and sulfotransferase
(SULT) $\}$ in hepatic systems were the predominant metabolic pathways responsible for the biotransformation of rhein. The fact that phase I (CYP 450) was weak in hepatic clearance reminds us to pay attention to the interaction of drugs especially that cleared only by CYP 450 when we put rhein into clinical application. Their experiment put forward the safety dose of rhein which can even be up to $600 \mathrm{mg}$ for 1 day [32]. To directly observe the pharmacokinetics of rhein in human body, Zhu et al. selected eight healthy male volunteers to join in a prospective crossover study. All subjects received a single dose of rhubarb extract $\left(50 \mathrm{mg} \cdot \mathrm{kg}^{-1}\right)$ on two separate occasions, once orally and once by a retention enema. Compared with retention enema administration, oral administration turned out to be more higher in the Cmax, AUC0- $\infty$, and AUMC, which means retention enema administration of rhein is the better choice for patients [33].

In a randomized controlled clinical trial, the patients with type- 2 diabetic nephropathy were treated by a rhein supplementation at the dose of oral $5 \mathrm{~g}$ per day for 2 months, 4 months, and 6 months. There was significant decrease of total cholesterol, triglycerides, and serum TGF- $\beta 1$ expression after 4-month and 6-month treatment [34]. Retention enema of rhein with other conventional treatments has also been proved to bring down proteinuria, blood urea nitrogen (BUN), and serum creatinine (Scr), attenuating the renal function patients, which means that rhein can be used as an adjuvant safe therapy for these patients [35]. Patients with overt DN who take orally a lecithinized formulation of rhein turned better through decreasing microglobulin blood beta 2 ( $\beta 2 \mathrm{MG})$, serum creatinine ( $\mathrm{Scr})$, serum cystatin $\mathrm{C}$ (CysC), and urinary albumin excretion rate (UAER) [36]. 


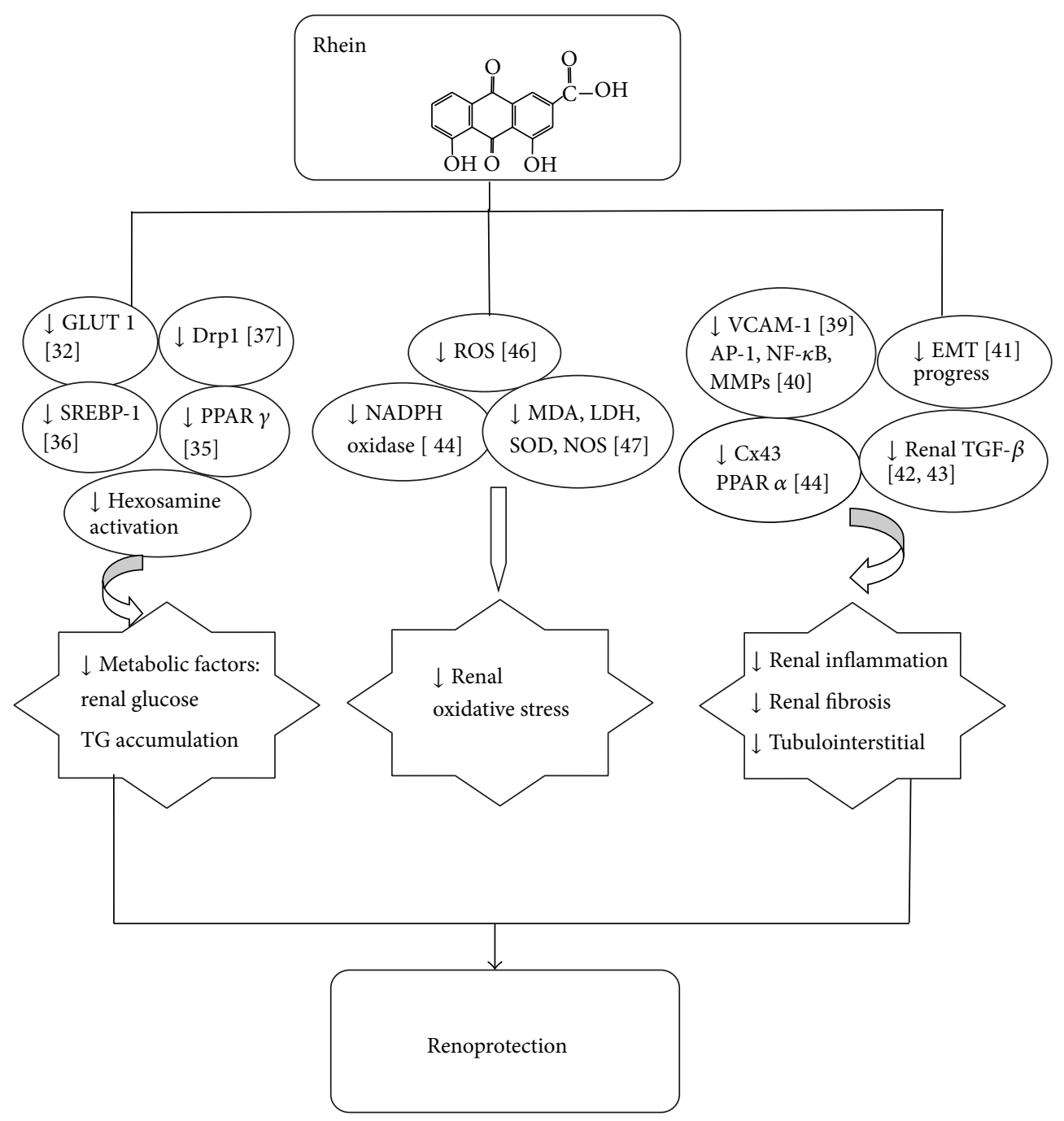

FIgURe 2: Possible mechanisms of rhein works on the renal protection.

\section{Conclusion}

A lot of scientific researches demonstrate that rhein has the ability to mediate multiple molecular targets, particularly oxidative stress and inflammation, which makes rhein a potential candidate for the therapy of some diseases, such as DN. In addition, rhein is characterized by mature extraction method, abundant resources, good tolerability, low price, and toxicity; thus, so many advantages can make it get more extensive application in clinical prevention and treatment in the future. Despite all of these big findings, more comprehensive and well randomized controlled human studies are still needed to explore.

\section{Conflict of Interests}

The authors declare that there is no conflict of interests regarding the publication of this paper.

\section{References}

[1] J. E. Shaw, R. A. Sicree, and P. Z. Zimmet, "Global estimates of the prevalence of diabetes for 2010 and 2030," Diabetes Research and Clinical Practice, vol. 87, no. 1, pp. 4-14, 2010.

[2] C. A. Phillips and M. E. Molitch, "The relationship between glucose control and the development and progression of diabetic nephropathy," Current Diabetes Reports, vol. 2, no. 6, pp. 523529, 2002.

[3] W. V. Brown, "Microvascular complications of diabetes mellitus: renal protection accompanies cardiovascular protection," The American Journal of Cardiology, vol. 102, no. 12, pp. 10L-13L, 2008.

[4] B. Grace, K. Hurst, and S. McDonald, "New patients commencing treatments in 2010," ANZDATA Registry Report 2011, Australia and New Zealand Dialysis and Transplant Registry, Adelaide, Australia, 2011.

[5] B. Puyanji, "Tannins pharmacological," Foreign Medical International, vol. 13, no. 5, pp. 19-22, 1991. 
[6] P. Xiao, L. He, and L. Wang, "Ethnopharmacologic study of chinese rhubarb," Journal of Ethnopharmacology, vol. 10, no. 3, pp. 275-293, 1984.

[7] Z.-H. He, M.-F. He, S.-C. Ma, and P. P.-H. But, "Anti-angiogenic effects of rhubarb and its anthraquinone derivatives," Journal of Ethnopharmacology, vol. 121, no. 2, pp. 313-317, 2009.

[8] M. Guo and H. Xu, "Progress in the pharmacological effects of Rhein," International Journal of Traditional Chinese Medicine, vol. 24, no. 3, pp. 139-143, 2002.

[9] X.-D. Cong, M.-J. Ding, D.-Z. Dai, Y. Wu, Y. Zhang, and Y. Dai, "ER stress, P66shc, and P-Akt/Akt mediate adjuvant-induced inflammation, which is blunted by argirein, a supermolecule and rhein in rats," Inflammation, vol. 35, no. 3, pp. 1031-1040, 2012.

[10] S. Y. Li, N. Jiang, S. S. Xie, K. D. G. Wang, X. B. Wang, and L. Y. Kong, "Design, synthesis and evaluation of novel tacrinerhein hybrids as multifunctional agents for the treatment of Alzheimer's disease," Organic and Biomolecular Chemistry, vol. 12, no. 5, pp. 801-814, 2014.

[11] K. Hao, Q. Qi, P. Wan et al., "Prediction of human pharmacokinetics from preclinical information of rhein, an antidiabetic nephropathy drug, using a physiologically based pharmacokinetic model," Basic and Clinical Pharmacology and Toxicology, vol. 114, no. 2, pp. 160-167, 2014.

[12] X. Zhao, J. Li, S. Zhu et al., "Rhein induces a necrosisapoptosis switch in pancreatic acinar cells," Evidence-Based Complementary and Alternative Medicine, vol. 2014, Article ID 404853, 7 pages, 2014.

[13] S. Dronavalli, I. Duka, and G. L. Bakris, "The pathogenesis of diabetic nephropathy," Nature Clinical Practice Endocrinology and Metabolism, vol. 4, no. 8, pp. 444-452, 2008.

[14] S. Yamagishi, K. Fukami, S. Ueda, and S. Okuda, "Molecular mechanisms of diabetic nephropathy and its therapeutic intervention," Current Drug Targets, vol. 8, no. 8, pp. 952-959, 2007.

[15] X. H. Guo, Z. H. Liu, A. Peng et al., "Rhein retards the progression of type 2 diabetic nephropathy in rats," Chinese Journal of Nephrology, vol. 18, no. 4, pp. 280-284, 2002 (Chinese).

[16] J.-M. Zheng, J.-M. Zhu, L.-S. Li, and Z.-H. Liu, "Rhein reverses the diabetic phenotype of mesangial cells over-expressing the glucose transporter (GLUT1) by inhibiting the hexosamine pathway," British Journal of Pharmacology, vol. 153, no. 7, pp. 1456-1464, 2008.

[17] S. Marshall, V. Bacote, and R. R. Traxinger, "Discovery of a metabolic pathway mediating glucose-induced desensitization of the glucose transport system: role of hexosamine biosynthesis in the induction of insulin resistance," Journal of Biological Chemistry, vol. 266, no. 8, pp. 4706-4712, 1991.

[18] Z.-H. Liu, Y.-J. Li, Z.-H. Chen, D. Liu, and L.-S. Li, “Glucose transporter in human glomerular mesangial cells modulated by transforming growth factor-beta and rhein," Acta Pharmacologica Sinica, vol. 22, no. 2, pp. 169-175, 2001.

[19] Y. Zhang, S. Fan, N. Hu et al., "Rhein reduces fat weight in $\mathrm{db} / \mathrm{db}$ mouse and prevents diet-induced obesity in $\mathrm{C} 57 \mathrm{Bl} / 6$ mouse through the inhibition of PPAR $\gamma$ signaling," PPAR Research, vol. 2012, Article ID 374936, 9 pages, 2012.

[20] X. Sheng, M. Wang, M. Lu, B. Xi, H. Sheng, and Y. Q. Zang, "Rhein ameliorates fatty liver disease through negative energy balance, hepatic lipogenic regulation, and immunomodulation in diet-induced obese mice," American Journal of Physiology: Endocrinology and Metabolism, vol. 300, no. 5, pp. E886-E893, 2011.
[21] J. Liu, Z. Chen, Y. Zhang et al., "Rhein protects pancreatic $\beta$ cells from dynamin-related protein-1-mediated mitochondrial fission and cell apoptosis under hyperglycemia," Diabetes, vol. 62, no. 11, pp. 3927-3935, 2013.

[22] H. Du, J. Shao, P. Gu, B. Lu, X. Ye, and Z. Liu, "Improvement of glucose tolerance by rhein with restored early-phase insulin secretion in db/db mice," Journal of Endocrinological Investigation, vol. 35, no. 6, pp. 607-612, 2012.

[23] G. Hu, J. Liu, Y.-Z. Zhen et al., "Rhein inhibits the expression of vascular cell adhesion molecule 1 in human umbilical vein endothelial cells with or without lipopolysaccharide stimulation," The American Journal of Chinese Medicine, vol. 41, no. 3, pp. 473-485, 2013.

[24] G. Martin, P. Bogdanowicz, F. Domagala, H. Ficheux, and J.P. Pujol, "Rhein inhibits interleukin- $\beta$-induced activation of MEK/ERK pathway and DNA binding of NF- $\kappa$ B and AP-1 in chondrocytes cultured in hypoxia: a potential mechanism for its disease-modifying effect in osteoarthritis," Inflammation, vol. 27, no. 4, pp. 233-246, 2003.

[25] L. Peng, J. Yang, C. Ning et al., "Rhein inhibits integrin-linked kinase expression and regulates matrix metalloproteinase9/tissue inhibitor of metalloproteinase-1 ratio in high glucoseinduced epithelial-mesenchymal transition of renal tubular cell," Biological and Pharmaceutical Bulletin, vol. 35, no. 10, pp. 1676-1685, 2012.

[26] Z. H. Jia, Z. H. Liu, J. M. Zheng, C. H. Zeng, and L. S. Li, "Combined therapy of rhein and benazepril on the treatment of diabetic nephropathy in $\mathrm{db} / \mathrm{db}$ mice," Experimental and Clinical Endocrinology and Diabetes, vol. 115, no. 9, pp. 571-576, 2007.

[27] Q. Gao, W.-S. Qin, Z.-H. Jia et al., "Rhein improves renal lesion and ameliorates dyslipidemia in $\mathrm{db} / \mathrm{db}$ mice with diabetic nephropathy," Planta Medica, vol. 76, no. 1, pp. 27-33, 2010.

[28] C. Hu, X. D. Cong, D.-Z. Dai, Y. Zhang, G. L. Zhang, and Y. Dai, "Argirein alleviates diabetic nephropathy through attenuating NADPH oxidase, Cx43, and PERK in renal tissue," NaunynSchmiedeberg's Archives of Pharmacology, vol. 383, no. 3, pp. 309-319, 2011.

[29] J.-R. Zong, Z.-M. Chao, Z.-L. Liu, and J. Wang, "Review about structure-function relationships of anthraquinone derivatives from Radix et Rhizoma Rhei," China Journal of Chinese Materia Medica, vol. 33, no. 20, pp. 2424-2427, 2008 (Chinese).

[30] S.-K. Heo, H.-J. Yun, E.-K. Noh, and S.-D. Park, "Emodin and rhein inhibit LIGHT-induced monocytes migration by blocking of ROS production," Vascular Pharmacology, vol. 53, no. 1-2, pp. 28-37, 2010.

[31] X.-F. Zhong, G.-D. Huang, T. Luo, Z.-Y. Deng, and J.-N. $\mathrm{Hu}$, "Protective effect of rhein against oxidative stress-related endothelial cell injury," Molecular Medicine Reports, vol. 5, no. 5, pp. 1261-1266, 2012.

[32] K. Hao, Q. Qi, P. Wan et al., "Prediction of human pharmacokinetics from preclinical information of rhein, an antidiabetic nephropathy drug, using a physiologically based pharmacokinetic model ," Basic and Clinical Pharmacology and Toxicology, vol. 114, no. 2, pp. 160-167, 2014.

[33] W. Zhu, X.-M. Wang, L. Zhang, X.-Y. Li, and B.-X. Wang, "Pharmacokinetic of Rhein in healthy male volunteers following oral and retention enema administration of rhubarb extract: a single dose study," The American Journal of Chinese Medicine, vol. 33, no. 6, pp. 839-850, 2005.

[34] Z. H. Xiong, "Effect of rhubarb on lipid and TGF- $\beta 1$ level in patients with diabetic nephropathy," Journal of Hainan Medical University, vol. 18, no. 8, pp. 1066-1068, 2012 (Chinese). 
[35] J. Guo, M. L. Chen, C. B. Chang et al., "Clinical analysis of diabetic nephropathy by rhubarb-based medicine enema," Clinical Focus, vol. 26, no. 18, pp. 1595-1598, 2011 (Chinese).

[36] X. Y. Xiao, R. Zhou, and F. S. Chen, "Clinical observation on treatment of 40 cases of early diabetic nephropathy by Niaoduqing granule," Journal of New Chinese Medicine, vol. 43, no. 8, pp. 48-49, 2011.

[37] M. Burke, M. R. Pabbidi, J. Farley et al., "Molecular mechanisms of renal blood flow autoregulation," Current Vascular Pharmacology, 2013.

[38] R. El-Baz, A. Settin, A. Ismaeel et al., "MTHFR C677T, A1298C and ACE I/D polymorphisms as risk factors for diabetic nephropathy among type 2 diabetic patients," Journal of the Renin-Angiotensin-Aldosterone System, vol. 13, no. 4, pp. 472477, 2012.

[39] D. G. Fogarty, J. C. Harron, A. E. Hughes, N. C. Nevin, C. C. Doherty, and A. P. Maxwell, "A molecular variant of angiotensinogen is associated with diabetic nephropathy in IDDM," Diabetes, vol. 45, no. 9, pp. 1204-1208, 1996.

[40] M. Eto, M. Saito, M. Okada et al., "Apolipoprotein E genetic polymorphism, remnant lipoproteins, and nephropathy in type 2 diabetic patients," The American Journal of Kidney Diseases, vol. 40, no. 2, pp. 243-251, 2002.

[41] J. M. Forbes, M. T. Coughlan, and M. E. Cooper, "Oxidative stress as a major culprit in kidney disease in diabetes," Diabetes, vol. 57, no. 6, pp. 1446-1454, 2008.

[42] Y. Wang, M. C. Y. Ng, S.-C. Lee et al., "Phenotypic heterogeneity and associations of two aldose reductase gene polymorphisms with nephropathy and retinopathy in type 2 diabetes," Diabetes Care, vol. 26, no. 8, pp. 2410-2415, 2003.

[43] S. K. Iyengar, H. E. Abboud, K. A. B. Goddard et al., "Genomewide scans for diabetic nephropathy and albuminuria in multiethnic populations: the Family Investigation of Nephropathy and Diabetes (FIND)," Diabetes, vol. 56, no. 6, pp. 1577-1585, 2007.

[44] M. B. Duran-Salgado and A. F. Rubio-Guerra, "Diabetic nephropathy and inflammation," World Journal of Diabetes, vol. 5, no. 3, pp. 393-398, 2014.

[45] M. Brownlee, "Biochemistry and molecular cell biology of diabetic complications," Nature, vol. 414, no. 6865, pp. 813-820, 2001.

[46] F. S. Yang and A. P. Yin, "Research progress about C-reactive protein and diabetic nephropathy," Medical Recapitulate, vol. 14, no. 3, pp. 432-434, 2008 (Chinese).

[47] K. M. Schmidt-Ott, "Unraveling the role of connective tissue growth factor in diabetic nephropathy," Kidney International, vol. 73, no. 4, pp. 375-376, 2008.

[48] T. L. Vasylyeva and R. J. Ferry Jr., "Novel roles of the IGF-IGFBP axis in etiopathophysiology of diabetic nephropathy," Diabetes Research and Clinical Practice, vol. 76, no. 2, pp. 177-186, 2007.

[49] S. Kume, D. Koya, T. Uzu et al., "Role of nutrient-sensing signals in the pathogenesis of diabetic nephropathy," BioMed Research International, vol. 2014, Article ID 315494, 9 pages, 2014.

[50] M. K. Diamond-Stanic, Y. H. You, and K. Sharma, "Sugar, Sex, and TGF- $\beta$ in Diabetic Nephropathy," Seminars in Nephrology, vol. 32, no. 3, pp. 261-268, 2012.

[51] N. Ishigaki, T. Yamamoto, Y. Shimizu et al., "Involvement of glomerular SREBP-1c in diabetic nephropathy," Biochemical and Biophysical Research Communications, vol. 364, no. 3, pp. 502508, 2007.
[52] D. Senthil, G. G. Choudhury, C. McLaurin, and B. S. Kasinath, "Vascular endothelial growth factor induces protein synthesis in renal epithelial cells: a potential role in diabetic nephropathy," Kidney International, vol. 64, no. 2, pp. 468-479, 2003. 


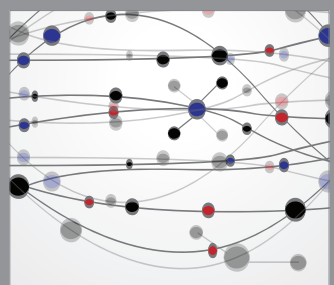

The Scientific World Journal
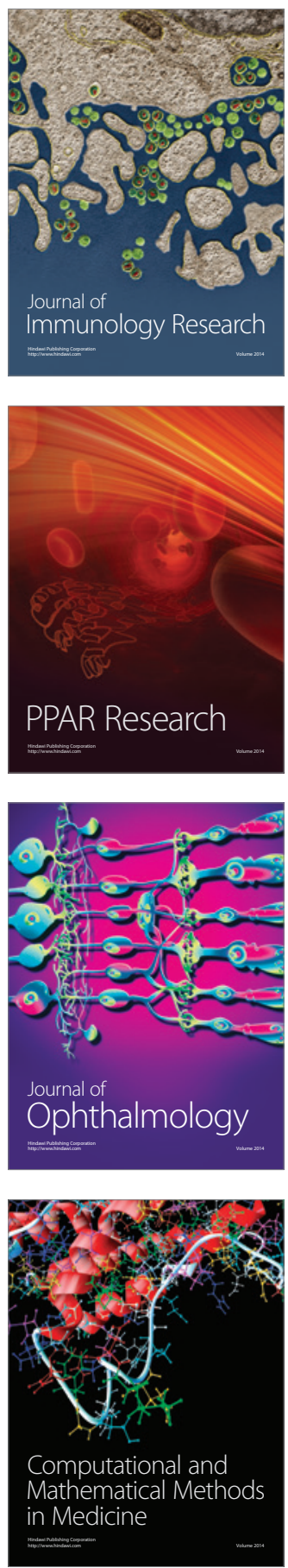

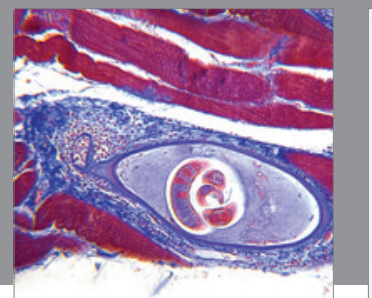

Gastroenterology

Research and Practice
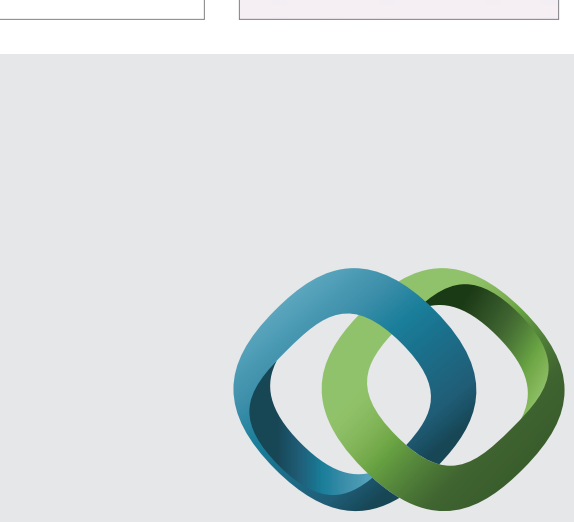

\section{Hindawi}

Submit your manuscripts at

http://www.hindawi.com
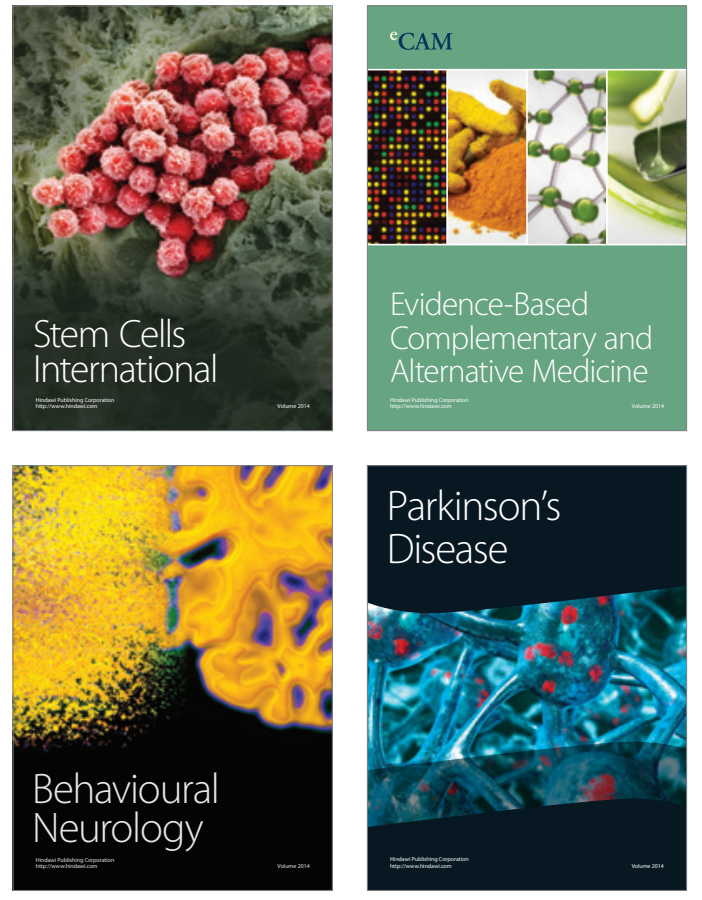
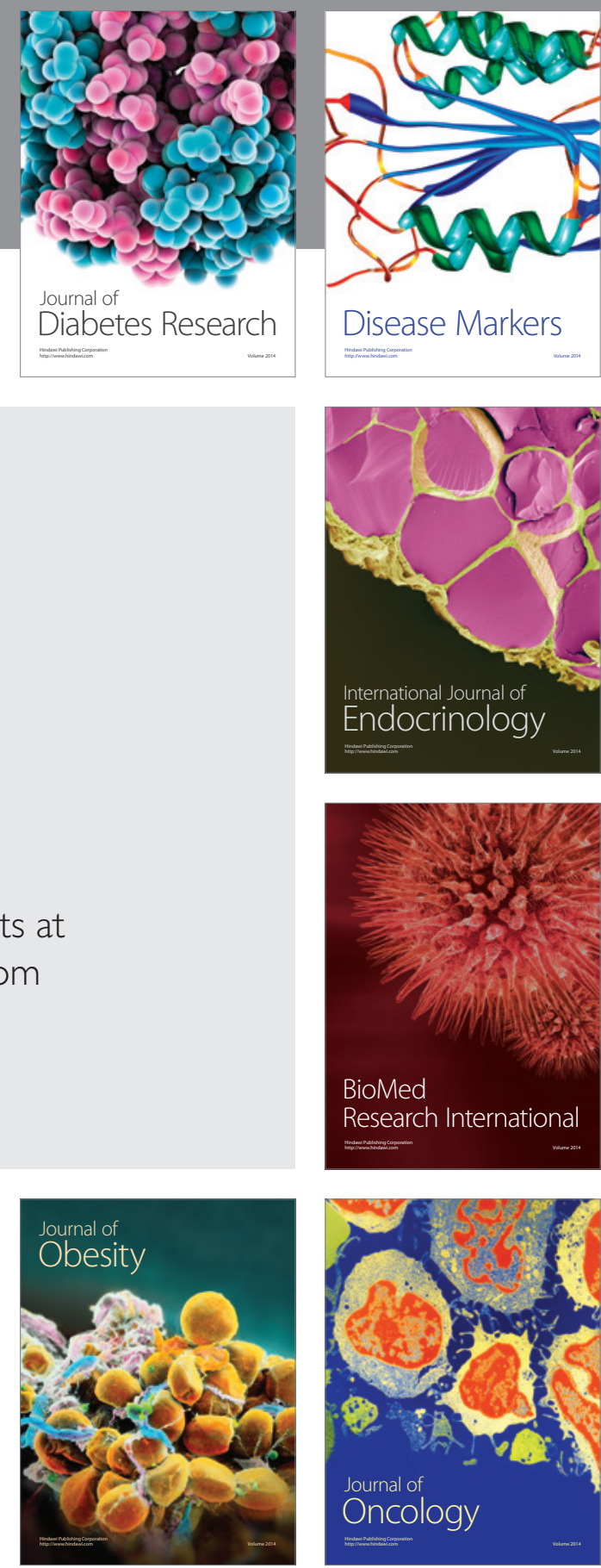

Disease Markers
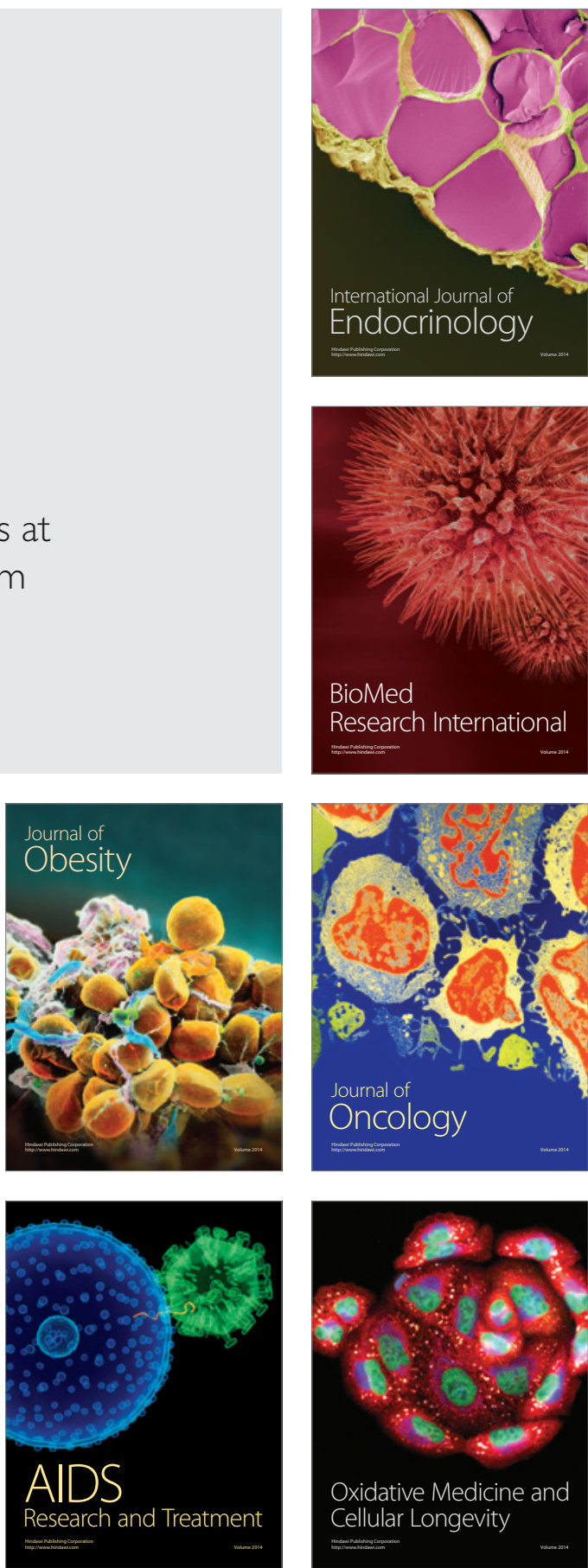